\title{
Pleuritis associated with immunoglobulin G4-related disease under normal thoracoscopic findings: a case report
}

\author{
Hiroki Shimada ${ }^{1}$, Yuto Kato ${ }^{1 *}$, Miyuki Okuda ${ }^{1}$, Koji Fukuda ${ }^{1}$, Nobuya Tanaka $^{1}$, Yutaro Okuda ${ }^{2}$ and \\ Akihiko Yoshizawa ${ }^{3}$
}

\begin{abstract}
Background: Immunoglobulin G4 (IgG4)-related disease is a chronic inflammatory disease that was recognized in 2011. Pleuritis associated with lgG4-related disease is rare and can be difficult to diagnose. Although there have been previous reports on pleuritis associated with lgG4-related disease by thoracoscopic findings, this is the first to observe pleuritis with lgG4-related disease from normal pleural thoracoscopic findings.

Case presentation: A 70-year-old Japanese female treated for breast cancer 33 years ago was referred to our hospital complaining of dyspnea on exertion. Chest computed tomography $(C T)$ revealed left pleural effusion that was exudative and predominant with lymphocytes, elevated adenosine deaminase (ADA) and Class III cytology (malignancy suspected). Subsequently, thoracoscopic pleural biopsy was performed for definitive diagnosis. Although pleural macroscopic findings appeared normal, we performed pleural biopsy at random sites. This patient was negative for mycobacterium tuberculosis, and neither granulomas nor malignant cells were found in the collected specimens. An infiltration of inflammatory cells, mainly plasma cells and lymphocytes, was observed. Immunostaining revealed the number of IgG4-positive plasma cells was 102/high power field (HPF), and the percentage of IgG4 positive/immunoglobulin G (IgG)-positive cells was 41.4\%. Since lgG4 serum levels were high and lgG4-related submandibular sialadenitis was also observed, a definitive diagnose of pleuritis associated with lgG4-related disease was confirmed.
\end{abstract}

Conclusions: We diagnosed pleuritis associated with lgG4-related disease by thoracoscopic pleural biopsy samples taken from a visually normal pleura. Although exudative pleural effusion with high ADA and lymphocyte predominance is a characteristic of tuberculous pleuritis, other diseases might be present. Since thoracoscopy can increase the diagnostic yield, pleural biopsy should be considered even if thoracoscopic pleural findings are deemed normal.

Keywords: IgG4-related disease, Medical thoracoscopy, Pleuritis

\section{Introduction}

Immunoglobulin G4 (IgG4)-related disease is a chronic inflammatory systemic disease that was recognized in 2011 [1]. IgG4-related disease, a multi-organ disease, is known to cause damage to the pancreas, bile duct,

*Correspondence: yklcab01kai02@gmail.com

${ }^{1}$ Hirakata Kohsai Hospital, 1-2-1, Fujisakahigashimachi, Hirakata, Osaka 573-0153, Japan

Full list of author information is available at the end of the article lacrimal gland, salivary gland, central nervous system, thyroid, lung, liver, digestive tract, kidney, prostate, retroperitoneum, artery, lymph node, skin, and mammary gland. Among these, lung lesions are particularly difficult to diagnose, requiring computed tomography (CT)-guided biopsy or thoracoscopy for diagnosis. On the other hand, reports of pleuritis associated with IgG4related diseases are rare, and only around 20 cases have thus far been reported. Pleural findings such as pleural plaque, fibrous deposits, pleural thickening, and nodules original author(s) and the source, provide a link to the Creative Commons licence, and indicate if changes were made. The images or other third party material in this article are included in the article's Creative Commons licence, unless indicated otherwise in a credit line to the material. If material is not included in the article's Creative Commons licence and your intended use is not permitted by statutory regulation or exceeds the permitted use, you will need to obtain permission directly from the copyright holder. To view a copy of this licence, visit http://creativecommons.org/licenses/by/4.0/. The Creative Commons Public Domain Dedication waiver (http://creativeco mmons.org/publicdomain/zero/1.0/) applies to the data made available in this article, unless otherwise stated in a credit line to the data. 
are recognized as IgG4-related disease, but currently, there are no reports on IgG4-related disease from normal plural findings assessed by thoracoscopy.

We report a case of pleuritis associated with IgG4related disease confirmed by randomly selected pleural biopsy sites after normal thoracoscopic findings.

\section{Case presentation}

A 70-year-old Japanese female complaining of dyspnea on exertion was admitted to our hospital after chest $\mathrm{X}$-ray showed left pleural effusion. Her medical history included hypertension, dyslipidemia, paroxysmal atrial fibrillation, cerebral infarction, and left breast cancer. Her hypertension, dyslipidemia, and paroxysmal atrial fibrillation were well managed by medication, and she underwent total left mastectomy and remnant gauze removal surgery 33 and 13 years ago, respectively. She was a nonsmoker with no history of autoimmune diseases, multiple myeloma, tuberculosis, or exposure to asbestos. Her family history revealed that her father had liver cirrhosis, and her mother had been diagnosed with lung cancer. There was no family history of autoimmune disease or tuberculosis.

On admission, she was lucid, with heart rate of 74 beats/minute, blood pressure of $138 / 68 \mathrm{mmHg}$, peripheral oxygen saturation of $96 \%$, and body temperature of $35.8{ }^{\circ} \mathrm{C}$. Heart sounds were normal but with decreased breath sounds in the left lung field. No spontaneous pain or tenderness was present in the chest. Her abdomen was flat and soft, with no abdominal tenderness or bowel sounds. No pitting edema was observed in either her forearms or legs; however, bilateral submandibular gland enlargement was observed. She had no motor or sensory deficits in her extremities and no arthralgia or morning stiffness associated with rheumatoid arthritis.

\section{Laboratory findings}

Inflammatory findings such as $\mathrm{C}$ reactive protein (CRP) and tumour makers were within the normal range (normal CRP, $<0.3 \mathrm{mg} / \mathrm{dL}$ ). Autoantibodies were serologically negative. Serum immunoglobulin G (IgG) was $2001 \mathrm{mg} /$ dL including $637 \mathrm{mg} / \mathrm{dL}$ for IgG4 (normal IgG 870$1700 \mathrm{mg} / \mathrm{dL}$, normal IgG4 4-108 mg/dL). Chest X-ray and CT showed left pleural effusion without abnormal lung shadow and no pleural thickening (Fig. 1a, b). We performed pleural puncture and drained $1200 \mathrm{~mL}$ of pleural fluid. Pleural puncture revealed lymphocytes were dominant and adenosine deaminase (ADA) was high, but Mycobacterium tuberculosis was not detected (normal ADA 8.6-20.5 U/L). Elevated IgG and IgG4 levels were observed in the pleural effusion (IgG $1756 \mathrm{mg} / \mathrm{dL}$; IgG4 $684 \mathrm{mg} / \mathrm{dL}$ ). Class III cells were identified from the pleural effusion, suggesting the possibility of breast cancer dissemination. Mycobacterium cultures for sputum, pleural fluid, and pleural biopsy samples were negative.

\section{Clinical progress}

The results of pleural puncture suggested tuberculous pleuritis, pleuritis due to autoimmune disease, or carcinomatous pleuritis, but a definitive diagnosis could not be made. Thus, we performed thoracoscopic pleural biopsy. Under thoracoscopy, no obvious lesions, pleural thickening, nodules, or pleural-pulmonary adhesions were found for the left pleura. Although the pleura appeared normal, we randomly collected seven specimens at three locations (Fig. 2).
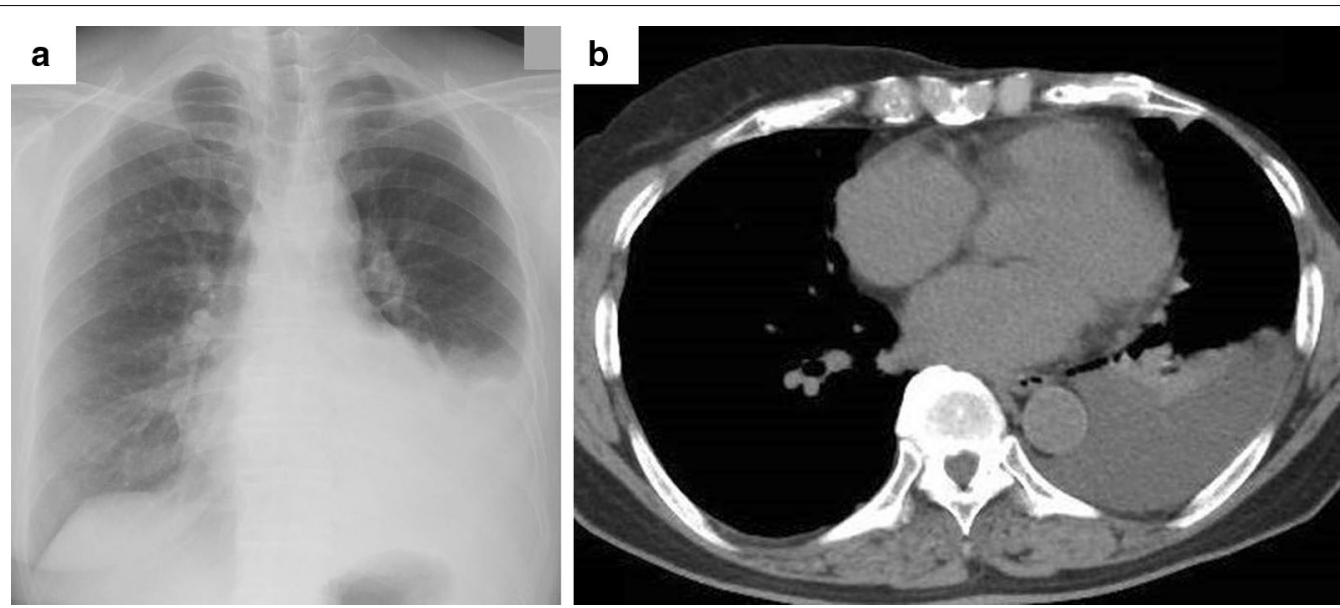

Fig. 1. Radiological findings. Chest $X$-ray at admission showed pleural effusion (a). Chest CT scan showed no thickening, calcification, or nodules in the left pleura. No tumour nodules were suspected in the lungs (b) 


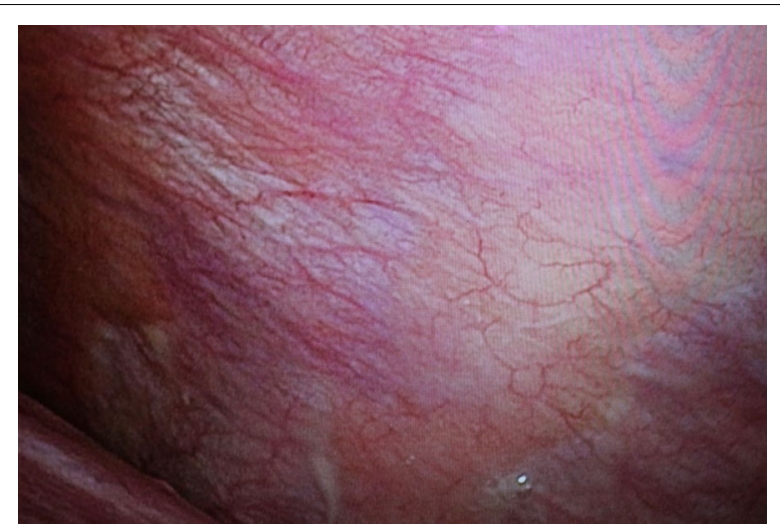

Fig. 2. Thoracoscopic findings of left pleura. Thoracoscopically, there were no obvious mass lesions, pleural thickening, nodules, or pleural-pulmonary adhesions observed for the left pleura

Pathological examination revealed membranous tissue with superficial proliferation in the reactive mesothelium. We confirmed plasmacytoid, lymphocyte-dominated inflammatory cell infiltration. The formation of reactive lymphatic follicles was also present. Polymerase chain reaction for mycobacterium tuberculosis (TB-PCR) was negative, and no granulomas were found in the pleural specimens. There were no malignant epithelial lesions and immunostaining showed no deviation in IgG-k or IgG- $\lambda$ chains. Epstein-Barr virus-encoded small Rino Nucleic Acid (RNA) and Congo red stain were also negative. IgG4-positive cells were 102/high power field (HPF), and the percentage of IgG4 positive/IgG positive cells was $41.4 \%$, suggesting pleuritis associated IgG4-related disease (Fig. 3a, b).

We then performed 2-deoxy-2-(18F]-fluoro-D-glucose (FDG) positron emission tomography (PET)-CT for systemic retrieval analysis. FDG PET-CT showed FDG accumulation within the bilateral submandibular glands of the left pleura. Subsequently, under ultrasound guidance, we collected biopsy samples from the right side of the submandibular gland. An infiltration of plasmacytes and lymphocytes around the adeno-atrial tissue was present; however, no malignant cells or granulomas were noted. Immunostaining confirmed IgG4-positive cells were $96 / \mathrm{HPF}$ and the percentage of IgG4 positive/IgG positive cells was $55.2 \%$, confirming pleuritis-associated IgG4-related disease.

We have started treatment with prednisolone $30 \mathrm{mg} /$ day because of increased pleural effusion after discharge from the hospital. The patient was followed for 10 months. The dose was reduced every month from 30 to $20 \mathrm{mg} /$ day to $15 \mathrm{mg} /$ day to $12.5 \mathrm{mg} /$ day to $10 \mathrm{mg} /$ day. Eventually, the dose was reduced to $7.5 \mathrm{mg} /$ day 6 months after the start of treatment, and now the dose was continued at $7.5 \mathrm{mg} /$ day. Since the prednisolone dose was reduced, the patient has progressed without relapse of pleuritis and submandibular gland swelling. Although some side effects of prednisolone such as lipid abnormalities and worsening of diabetes were observed, the treatment was successful without major side effects.

\section{Discussion}

In this case, a biopsy from a grossly normal pleura led to the diagnosis of pleuritis associated with IgG4-related disease, and remission was achieved with steroid therapy. Although papers on IgG4-related diseases have reported abnormal findings in the pleura [2-4], we were able to confirm a definitive diagnosis of pleuritis associated with IgG4-related disease by performing random biopsies of the pleura using thoracoscope. IgG4-related disease is a chronic inflammatory condition characterized by mass

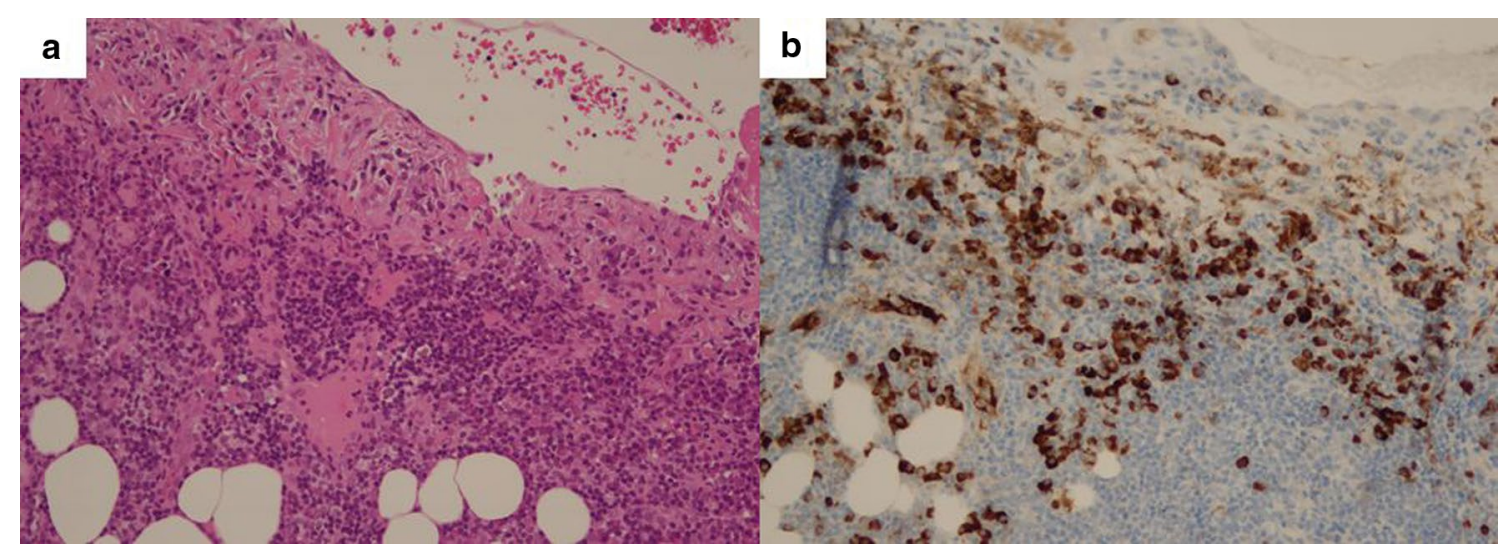

Fig. 3. Pathological findings of the left pleura. Biopsy specimens stained with hematoxylin and eosin stain (a) and lgG4 immunostaining (b). Plasmacytoid, lymphocyte-dominated inflammatory cell infiltration was present. The formation of reactive lymphatic follicles was also found 
or hypertrophic lesion infiltration with lymphocytes and IgG4-secreting plasma cells with various degrees of fibrosis. The clinical diagnostic criteria for IgG4-related disease states: (1) clinical examination showing characteristic diffuse/localized swelling or masses in single/multiple organs; (2) hematological examination of elevated serum IgG4 concentrations (> $135 \mathrm{mg} / \mathrm{dL}$ ); (3) histopathologic examination showing (a) marked lymphocyte and plasmacyte infiltration and fibrosis and (b) infiltration of IgG4-positive plasma cells: ratio of IgG4positive/IgG positive cells > 40\% and > 10 IgG4-positive plasma cells/HPF [5]. This present case satisfied all of the aforementioned criteria.

Additionally, the diagnostic criteria for IgG4-related respiratory disease were proposed as follows: (1) abnormal shadow on chest CT; (2) serum IgG4 level of $>135 \mathrm{mg} / \mathrm{dL}$; (3) histopathologic features fulfilling the comprehensive diagnostic criteria; (4) presence of extrathoracic lesions [6]. IgG4-related diseases can cause interstitial pneumonia, inflammatory nodules, and airway inflammation, but pleuritis is uncommon.

Pleural effusion for heart and renal failures is often due to systemic diseases associated with leakage or bilateral pleural effusion. Pleural effusion due to IgG4-related disease is expected to be exudative pleural effusion due to inflammation and can take the form of either unilateral or bilateral pleural effusion patterns. Pleural effusion in pleuritis associated with IgG4-related disease has been reported to be lymphocyte-dominated exudative pleural effusion with high ADA [7]. To date, lymphocyte-dominated exudative pleural effusion with a high level of ADA has been considered an indication of tuberculous pleuritis. A report on ADA [8] showed a sensitivity of $100 \%$ and a specificity of $93.9 \%$ for tuberculous pleuritis if the cutoff value of ADA was set for $40.3 \mathrm{U} / \mathrm{L}$, after which, many studies have shown the diagnostic accuracy of tuberculous pleuritis to be high $[9,10]$. To diagnose tuberculous pleuritis, it is important to confirm by mycobacterium culture and TB-PCR. Other diagnostic tools are cell fractionation of the pleural fluid, pleural fluid ADA activity, QuantiFERON TB-2G (QFT-2G; Cellestis Ltd., Carnegie, Victoria, Australia), and purified protein derivative (PPD) reaction.

However, Miyoshi et al. reported that only 1 of 32 cases of tuberculous pleuritis had a positive pleural fluid culture, and it is thought that the diagnosis based on pleural fluid examination alone is extremely difficult. Pleural biopsy, which was performed in this case, is said to be useful for diagnosis and has superior sensitivity and specificity compared to pleural fluid examination [11]. In tuberculous pleuritis, pleural gross findings often show nodular lesions and pleural thickening, and pathology proves butyric granulomas. Tuberculous pleuritis was considered negative since this case showed elevated ADA but no evidence of mycobacterium tuberculosis on pleural fluid culture or pleural biopsy sample, and the gross findings of pleura were normal. Pathology did not reveal butyric granulomas, and her TB-PCR was negative.

On the other hand, exudative pleural effusion with high ADA and lymphocyte-dominated pleural effusion is not a finding specific to tuberculous pleuritis since it is also found in primary effusion lymphoma, leukemia, pyothorax, rheumatic disease, and pleural effusion associated with malignant pleural mesothelioma. It has been reported that thoracoscopic pleural biopsy can provide a definitive diagnosis $90 \%$ of the time $[12,13]$. Therefore, for our case, a diagnosis of pleuritis associated IgG4-related disease obtained from pleural biopsy is considered quite accurate.

Thus far, three patterns of thoracoscopic findings have been described for IgG4-associated pleuritis: (1) milky pleural plaques suggestive of hyalinized collagen fiber deposits; (2) diffuse inflammatory thickening of the pleura; (3) nodules on the parietal pleura [2]. In each of these cases, pleural biopsies showed an infiltration of IgG4-positive cells. However, in our case, the thoracoscopic findings were normal, but random biopsies led to a definitive diagnosis. Pleural biopsy is considered useful for diagnosis and should be performed even when thoracoscopic findings of the pleura appear normal.

\section{Conclusions}

Although thoracoscopic findings of the pleura appeared normal in this case, we performed random pleural biopsies to confirm a diagnosis of pleuritis associated with IgG4-related disease. We suggest that thoracoscopic pleural biopsy should be considered even when pleural findings on thoracoscopy appear normal.

\section{Abbreviations}

IgG4: Immunoglobulin G4; IgG: Immunoglobulin G; CT: Computed tomography; ADA: Adenosine deaminase; FDG: 2-Deoxy-2-[18F]-fluoro-D-glucose; PET: Positron emission tomography; HPF: High power field; TB-PCR: Polymerase chain reaction for Mycobacterium tuberculosis; QFT-2G: QuantiFERON TB-2G; PPD: Purified protein derivative.

\section{Acknowledgements}

We would like to thank our patient and her family.

\section{Authors' contributions}

The manuscript was prepared by HS under the supervision of YK and MO. Pleural biopsy was performed by HS, YK and NT. Immunostaining was performed by AY. All authors read and approved the final manuscript.

\section{Funding}

The authors declare that this study was not funded. 


\section{Availability data and materials}

The authors declare that the data supporting the findings of this case report are available within the article and its additional information files.

\section{Ethics approval and consent to participate}

The Ethics Committee at Hirakata Kohsai Hospital waived the need for approval.

\section{Consent for publication}

Written informed consent was obtained from the patient for publication of this case report and any accompanying images. A copy of the written consent is available for review by the Editor-in-Chief of this journal.

\section{Competing interests}

The authors declare no competing interests.

\section{Author details}

${ }^{1}$ Hirakata Kohsai Hospital, 1-2-1, Fujisakahigashimachi, Hirakata, Osaka 573-0153, Japan. ${ }^{2}$ Department of Respiratory Medicine, Nara Medical University Hospital, 840 Shijocho, Kashihara, Nara 634-8522, Japan. ${ }^{3}$ Department of Diagnostic Pathology, Kyoto University Hospital, 54 Shougoin-Kawaharacho, Sakyo-ku, Kyoto 606-8507, Japan.

Received: 19 July 2020 Accepted: 3 February 2021

Published online: 30 April 2021

\section{References}

1. Stone JH, Khosroshahi A, Deshpande V, Chan JKC, Heathcote JG, Aalberse R, et al. Recommendations for the nomenclature of IgG4-related disease and its individual organ system manifestations. Arthritis Rheum. 2012;64(10):3061-7.

2. Yasokawa N, Shirai R, Tanaka H, Kurose K, Oga T, Oka M. Thoracoscopic findings in lgG4-related pleuritis. The Japanese Society of Internal Medicine. Intern Med. 2020;59(2):257-60.
3. Tong X, Bai M, Wang W, Han Q, Tian P, Fan H. IgG4-related disease involving polyserous effusions with elevated serum interleukin-6 levels: a case report and literature review. Immunol Res. 2017;65(4):944-50.

4. Ramponi S, Gnetti L, Marvisi M. Lung manifestations of IgG4- related disease. A multifaceted disorder. Sarcoidosis Vasc Diff. 2018;35(1):74-80.

5. Umehara H, Okazaki K, Masaki Y, Kawano M, Yamamoto M, Saeki T, et al. Comprehensive diagnostic criteria for lgG4-related disease (IgG4-RD), 2011. Mod Rheumatol. 2012;22(1):21-30.

6. Matsui S, Yamamoto H, Minamoto S, Waseda Y, Mishima M, Kubo K. Proposed diagnostic criteria for lgG4 related respiratory disease. Respir Investig. 2016;54(2):130-2.

7. Nagayasu A, Kubo S, Nakano K, Nakayamada S, et al. IgG4-related pleuritis with elevated adenosine deaminase in pleural effusion. Intern Med. 2018:57(15):2251-7.

8. Krenke R, Safianowska A, Paplinska M, Krenke R, et al. Pleural fluid adenosine deaminase and interferon gamma as diagnostic tools in tuberculosis pleurisy. J Physiol Pharmacol. 2008;59(Suppl 6):349-60.

9. Ocana I, Martinez-Vazquez JM, Segura RM, Fernandez-De-Sevilla T, Capdevila JA. Adenosine deaminase in pleural fluids. Test for diagnosis of tuberculous pleural effusion. Chest. 1983:84(1):51-3.

10. Valdes L, Alvarez D, San Jose E, et al. Tuberculous pleurisy: a study of 254 patients. Arch Intern Med. 1998;158(18):2017-21.

11. Miyoshi M, Fukushima F, Takizawa H, et al. Usefulness of thoracoscopy under local anesthesia in tuberculous pleuritis. Dokkyo J Med Sci. 2012:39(3):193-9.

12. Blanc F, Atassi K, Bignon J, et al. Diagnostic value of medical thoracoscopy in pleural disease: a 6-year retrospective study. Chest. 2002;121(5):1677-83.

13. Sugiyama $M$, Horiguchi $T$, Ishibashi $A$, et al. Clinical utility and safety of diagnostic thoracoscopy. Ann Jpn Respir Soc. 2001;39(12):899-902.

\section{Publisher's Note}

Springer Nature remains neutral with regard to jurisdictional claims in published maps and institutional affiliations.
Ready to submit your research? Choose BMC and benefit from:

- fast, convenient online submission

- thorough peer review by experienced researchers in your field

- rapid publication on acceptance

- support for research data, including large and complex data types

- gold Open Access which fosters wider collaboration and increased citations

- maximum visibility for your research: over $100 \mathrm{M}$ website views per year

At $\mathrm{BMC}$, research is always in progress.

Learn more biomedcentral.com/submissions 